\title{
ASPECTS OF LASER LORENZ DYNAMICS
}

\author{
P. B. CHAPMAN ${ }^{1}$
}

(Received 5 March 1992; revised 20 October 1992)

\begin{abstract}
The laser Lorenz equations are studied by reducing them to a form suitable for application of an extension of a method developed by Kuzmak. The method generates a flow in a Poincare section from which it is inferred that a certain Hopf bifurcation is always subcritical.
\end{abstract}

\section{Introduction}

A version of the complex Lorenz equations models the behaviour of certain lasers, and an extensive discussion of the background to this model may be found in the text by Weiss and Vilaseca [8]. The theoretical studies of the original complex Lorenz equations made by Fowler, Gibbon and McGuinness [4], and by Fowler and McGuinness [5] contain results that may not be relevant to the laser case - see the remark by Zeghlache et al. [9]. Although they have many of the properties of the "complex Lorenz" equations, the "laser Lorenz" equations studied below are more tractable than the former.

The present method is distinct from that followed by Fowler and McGuinness [5]. Here the first step is to reduce the fifth order laser Lorenz equations to effectively fourth order by choosing a new set of variables, and this is possible in the case of major interest when a physical parameter $A$ (the pump parameter) exceeds a critical value $A_{\text {crit }}$, necessarily greater than unity. The physical variables are scaled with respect to the quasi-steady solutions (in fact supercritical, Hopf bifurcated solutions which are now designated as primary) that become available when ( $A-A_{\text {crit }}$ ) is positive. Then a new choice of variables produces a fourth order system, which for large $\left(\left(A-A_{\text {crit }}\right) \propto \epsilon^{-2}\right)$ can be viewed as essentially an $\epsilon$-perturbed, nonlinear, oscillatory second order system. The reduction, which is exact, together with its associated local, fixed point linear dynamics is set out in Section 2. The nonlinear dynamics of the reduced system are studied in the subsequent ones.

${ }^{1}$ Department of Mathematics, University of Western Australia, Nedlands, W.A., 6009

(C) Australian Mathematical Society, 1994, Serial-fee code 0334-2700/94 
In Section 3 solutions of the underlying $(\epsilon=0)$ steady nonlinear oscillator are constructed. The description appears to be the simplest possible with the ultimate computation in mind and proceeds by (fairly) direct computation of the Fourier representation of the oscillations, suitably parameterized, rather than by appealing to appropriate but less convenient special functions. The coefficients of the Fourier representation are infinite series in the describing parameter $\Delta$, with non-small common convergence radius. They can be calculated to arbitrary powers of $\Delta$ but the effort required increases very rapidly with the index.

With such a description of the underlying oscillator, an extended form of Kuzmak's [7] method applies to the perturbed $(\epsilon \neq 0)$ system. The present analytic framework differs from that adopted by Bourland and Haberman [1], who also use essentially Kuzmak's method in their study of a nonlinear oscillator, but the basic ideas are the same. In Section 4 a solution matrix and its inverse are found for the linear homogeneous equations of first variation for the unperturbed system. All the entries in these matrices can be directly expressed in terms of the Fourier representations described earlier. Then, in Section 5, Kuzmak's [7] idea is used: conditions are specified whereby the slow evolution of parameters determining the underlying oscillator's Fourier representation forces the solution of the non-homogeneous first variation system to be periodic in a fast variable. These calculations are presented in a general and systematic framework, and they lead to a first order equation whose direction field can be interpreted as an abstract Poincaré section of the trajectories of the fourth order system.

Calculations (Section 6) elucidate some of the properties of this field. Most significantly it is found that Hopf bifurcation of the reduced system, designated as secondary, is always subcritical. This result distinguishes the laser Lorenz system from the complex Lorenz system, which (Fowler, Gibbon and McGuinness [4]) may show both suband supercritical Hopf bifurcations in certain regions in parameter space. A second result is that the toroidal frequency of all oscillatory solutions asymptotically tends to that $(\omega)$ of the primary Hopf bifurcation solutions.

The writer is unaware of any proof of validity of approximations obtained with Kuzmak's technique. (The original paper [7] contains a proof that the residual is suitably small but this, while suggestive, is not quite the same thing.) An outline of a proof is given in Section 7.

\section{The laser Lorenz dynamical system}

The dynamical system studied is (Zeghlache et al. [9])

$$
\begin{aligned}
& \dot{E}=-\kappa(1+i \delta) E-\kappa A P, \\
& \dot{P}=-\gamma_{\perp}(1+i \hat{\Delta}) P-\gamma_{\perp} D E,
\end{aligned}
$$




$$
\dot{D}=-\gamma_{\|}(D-1)+\gamma_{\|} \mathcal{R} \ell\left(P^{*} E\right) \text {, }
$$

where $\kappa, A, \gamma_{\perp}$ and $\gamma_{\|}$are positive constants; $\delta$ and $\hat{\Delta}$ are also real constants but they may have either sign. The independent variable is $t$ and differentiation with respect to it is denoted by . The dependent variables $E$ and $P$ are complex, while $D$ is real. Complex conjugation is denoted by * . The theory will be directed towards describing the behaviour of the system when the parameter $A$ is large, with scaling imposed by bifurcation dynamics.

There is a singular point of the system (2.1-3) at $E=P=0, D=1$. Here its local linear approximation has a coefficient matrix with one real eigenvalue $\left(-\gamma_{\|}\right)$exactly, and a quartet of eigenvalues which are generally complex. For "small $A$ " they are approximately

$$
-\kappa(1 \pm i \delta)+O(A), \quad-\gamma_{\perp}(1 \pm i \Delta)+O(A)
$$

in the "large $A$ " case they are approximately

$$
\sqrt{\gamma_{\perp} \kappa A}+O(1), \quad-\sqrt{\gamma_{\perp} \kappa A}+O(1)
$$

and their conjugates, that is, complex numbers whose arguments are close to zero or $\pi$. This follows from the usual calculations, and the change in sign of the real part of one of the pair signals a supercritical Hopf bifurcation with $A$ the bifurcation parameter.

These primary Hopf bifurcation solutions exist when $A$ exceeds a critical value $A_{\text {crit }}$ and they are the quasi-steady solutions

$$
\begin{aligned}
& E(t)=E_{L}(t)=E_{0} e^{i \omega t}, \\
& P(t)=P_{L}(t)=P_{0} e^{i \omega t}, \\
& D(t)=D_{0},
\end{aligned}
$$

where $D_{0}$ and $\omega$ are real constants, and $E_{0}$ and $P_{0}$ are generally complex constants (that is, independent of $t$ ) determined to within a unimodular, constant factor corresponding to a choice of $t$ origin. Thus, without significant loss of generality one of them, $P_{0}$ say, can be taken to be real and positive

$$
\arg \left(P_{0}\right)=0
$$

It follows that such solutions require

$$
\omega=-\kappa \gamma_{\perp}(\hat{\Delta}+\delta) /\left(\kappa+\gamma_{\perp}\right),
$$


- notably, $\omega$ has no $A$ dependence - and the remaining real constant $D_{0}$ to be determined from the relation

$$
\begin{aligned}
\mathcal{R} \ell\left(P_{0} E_{0}^{*}\right) & \equiv H=D_{0}-1 \\
& =\left(\left(\kappa \delta-\gamma_{\perp} \hat{\Delta}\right)^{2}+(1-A)\left(\kappa+\gamma_{\perp}\right)^{2}\right) /\left(A\left(\kappa+\gamma_{\perp}\right)^{2}\right) \\
& \equiv-1+A_{\text {crii }} / A,
\end{aligned}
$$

which incidentally defines the positive parameter $A_{\text {crit }}$. As the ratio

$$
E_{0} / P_{0}=-\kappa A /(\kappa+i(\kappa \delta+\omega))
$$

is fixed from (2.1), then because

$$
P_{0} E_{0}^{*}=P_{0}^{2} E_{0}^{*} / P_{0},
$$

it follows that $H$ must be negative in order that the quasi-steady solutions can exist, in fact

$$
H=-P_{0}^{2} \kappa^{2} A /\left(\kappa^{2}+(\kappa \delta+\omega)^{2}\right) .
$$

This requires that the earlier (2.9) evaluation of $H$ must also be negative, and its sign is so determined only for sufficiently large

$$
A>A_{\text {crit }}=1+\left(\kappa \delta-\gamma_{\perp} \hat{\Delta}\right)^{2} /\left(\kappa+\gamma_{\perp}\right)^{2} \geq 1 .
$$

When this condition is met, then the magnitude of $P_{0}$ can be calculated from (2.12)

$$
P_{0}^{2}=-H\left(\kappa^{2}+(\kappa \delta+\omega)^{2}\right) /\left(\kappa^{2} A\right)
$$

and with $\arg \left(P_{0}\right)$ chosen to be zero, (2.10) determines $E_{0}$.

The eigenvalues of the system obtained by linearization about the quasi-steady solution (2.5-7), using substitutions

$$
E=E_{L}(1+x), \quad P=P_{L}(1+y), \quad D=D_{0}+d,
$$

are zero (exactly); two real and negative

$$
\lambda_{1}=-\left(\kappa+\gamma_{\perp}\right)+O\left(A^{-1}\right), \quad \lambda_{2}=-2 \kappa+O\left(A^{-1}\right) ;
$$

and a complex conjugate pair which are expressible as

$$
\lambda_{3,4}= \pm i \sqrt{\gamma_{\perp} \gamma_{\|} A|H|}+\left(\kappa-\gamma_{\perp}-\gamma_{\|}\right) / 2+O\left(A^{-1 / 2}\right)
$$

when $\left(A-A_{\text {crit }}\right)=A|H|$ is sufficiently large. So, asymptotically at least, the hyperplane $\left(\kappa-\gamma_{\perp}-\gamma_{\|}\right)=0$ in parameter space is a stability boundary for the quasisteady solutions (2.5-7), and experience suggests that interesting dynamics could occur in its neigbourhood. 
The facts that the (fifth order in real variables) perturbation system from the quasisteady state obtained from (2.1-3) is autonomous, and its linearization has a zero eigenvalue, suggest that the problem can be reduced to an effectively fourth order one in real variables. As well, the "large $A$ " requirement for instability makes a perturbation theory a natural approach.

With these thoughts in mind, proceed as follows. As a preliminary move, scale with respect to the primary Hopf bifurcated solutions by setting

$$
E=E_{L} U ; \quad P=P_{L} V ; \quad D=D_{0}+d
$$

in (2.1-3), reduce them, and then introduce a scaled variable

$$
\tau=\epsilon^{-1} t
$$

with the perturbation (small) parameter

$$
\epsilon=(|H| A)^{-1 / 2}=\left(A-A_{\text {crit }}\right)^{-1 / 2} .
$$

(In these variables, similarities with and differences from the complex Lorenz equations $[4,5]$ are evident.) Denote the derivative with respect to $\tau$ with $\mathbf{a}^{\prime}$, and eliminate $V$ from the system by replacing it with a new variable

$$
W=U^{\prime} \text {. }
$$

After expressing $U$ in polar form

$$
U=R e^{i \Psi}
$$

with $R$ and $\Psi$ both real functions of $\tau$, it transpires that the resulting real equations can all be expressed with a variable

$$
T=R^{2} \Psi^{\prime}
$$

instead of $\Psi$, and so the effective order of the system is reduced by one in variables $R, T, d$ and

$$
S=R^{\prime} \text {. }
$$

This last system can then be reformulated as a weakly fourth order, essentially perturbed, second order one by replacing the variable $d$ with

$$
v=|H|^{-1} d+\gamma_{\|} R^{2} /(2 \kappa) .
$$

Finally, it is an advantage to replace the variables $R$ and $S$ with the variables

$$
\begin{gathered}
r=R^{2} \geq 0, \\
s=r^{\prime}=2 R R^{\prime}, \quad S=r^{\prime} /\left(2 r^{1 / 2}\right) .
\end{gathered}
$$


Then the final system reduces to (2.21), now

$$
\Psi^{\prime}=T / r
$$

which can be integrated separately using solutions of the system

$$
\begin{aligned}
r^{\prime} & =s, \\
s^{\prime} & =\left(s^{2}+4 T^{2}\right) /(2 r)-\epsilon(p s-2 q T)+2 \kappa \gamma_{\perp}\left(v-\gamma_{\|} r /(2 \kappa)\right) r, \\
T^{\prime} & =-\epsilon(p T+q s / 2), \\
v^{\prime} & =-\epsilon \gamma_{\|}\left(v-1+\left(1-\gamma_{\|} /(2 \kappa)\right) r\right),
\end{aligned}
$$

obtained on invoking relations among the $E_{0}$ and $P_{0}$. Here parameter combinations

$$
p=\kappa+\gamma_{\perp}
$$

and

$$
\begin{aligned}
q & =\kappa \delta+\gamma_{\perp} \hat{\Delta}+2 \omega \\
& =\left(\kappa-\gamma_{\perp}\right)\left(\kappa \delta-\gamma_{\perp} \hat{\Delta}\right) /\left(\kappa+\gamma_{\perp}\right)
\end{aligned}
$$

are introduced, and they are independent of $A$. It is emphasised that the reduction to (2.25-28) is exact. (The corresponding reduction in [5] is evidently approximate; see $2.8_{3}$ in that study.) Properties of the special case $\gamma_{\|}=2 \kappa$, in which the last equation (2.28) of the system decouples, are studied by Flessas [3] (without recourse to the reduction described above), so his results are not placed in a general context.

Singular points of the reduced system occur at

$$
\mathrm{C}:(r, s, T, v)=(0,0,0,1)
$$

(corresponding to $E=P=0, D=1$ ) and

$$
\text { D : }(r, s, T, v)=\left(1,0,0, \gamma_{\|} / 2 \kappa\right) \text {. }
$$

It will be seen below that the possibly suspect procedure of local linearization of the system (2.25-28) at the point $\mathbf{C}$ can be justified. If it is done (ignoring the singular term $\left(s^{2}+4 T^{2}\right) /(2 r)$ in $\left.(2.26)\right)$, it will be found that for small $\epsilon$ the eigenvalues of the linearization are all real; two of unit order

$$
\pm \sqrt{2 \kappa \gamma_{\perp}}+O(\epsilon)
$$

and two small and negative,

$$
-\epsilon \gamma_{l} \text { and }-\epsilon p+O\left(\epsilon^{3}\right) .
$$


The eigenvalues at the point $\mathbf{D}$ have in essence been stated (2.14-15); in the present scaling and when $\epsilon$ is small there are two small real ones

$$
-2 \epsilon \kappa+O\left(\epsilon^{3}\right) \text { and }-\epsilon p+O\left(\epsilon^{3}\right)
$$

and a complex conjugate pair

$$
\pm i \sqrt{\gamma_{\|} \gamma_{\perp}}+\epsilon\left(\kappa-\gamma_{\|}-\gamma_{\perp}\right) / 2+O\left(\epsilon^{2}\right) .
$$

When $\epsilon$ is large, that is when $\left(A-A_{\text {crit }}\right)$ is small but positive, the real eigenvalues have negative real parts. The real ones are

$$
-\gamma_{\|} \epsilon+O\left(\epsilon^{-1}\right) \text { and } \quad-2 \kappa \gamma_{\perp} p /\left(\left(p^{2}+q^{2}\right) \epsilon\right)+O\left(\epsilon^{-3}\right)
$$

(one large, one small), and the complex conjugate pair are

$$
-(p \pm i q) \epsilon+O\left(\epsilon^{-1}\right) \text {. }
$$

Equation (2.33) suggests, and it can be shown that for small enough $\epsilon$ (large enough $\left.A-A_{\text {crit }}\right)$ a necessary and sufficient condition for the real part of some eigenvalue to become positive is

$$
\left(\kappa-\gamma_{\perp}-\gamma_{\|}\right)>0,
$$

and it can also be shown that a sufficient condition for no eigenvalue to have a positive real part is

$$
\left(\kappa-\gamma_{\perp}-\gamma_{\|}\right)<0
$$

for any non-negative $\epsilon$. It can also be inferred that there is at most one boundary crossing possible as $\epsilon$ decreases (that is, for $A-A_{\text {crit }} \in[0, \infty)$ ), so just one consequent secondary bifurcation is indicated.

The aim is now to investigate the dynamics of the secondary bifurcation by approximating solutions of equations (2.25-28) for small $\epsilon$ on intervals of the independent variable of extent greater than $O\left(\epsilon^{-1}\right)$, using what is effectively a generalization of the method proposed by Kuzmak [7] for problems involving a restricted class of perturbation to otherwise autonomous oscillators.

\section{The unperturbed system}

When $\epsilon=0$ the system $(2.25-28)$ is

$$
\begin{aligned}
r^{\prime} & =s, \\
s^{\prime} & =\left(s^{2}+4 T^{2}\right) /(2 r)+2 \kappa \gamma_{\perp}\left(v-\gamma_{\|} r /(2 \kappa)\right) r \\
T^{\prime} & =v^{\prime}=0 .
\end{aligned}
$$


Suitable descriptions of solutions of this system are central to the application of Kuzmak's method. Obviously, two components of its solution vector are

$$
T=T_{0} \quad \text { and } \quad v=v_{0},
$$

both constant. These solutions force all phase trajectories $r(s)$ of $(3.1-2)$ to be closed (that is, $r(\tau)$ to be oscillatory). For consider the equation

$$
\left(r^{\prime}\right)^{2}=2 L r-4 T_{0}^{2}+4 a r^{2}-2 b r^{3},
$$

where $L, T_{0}, a$ and $b$ are constants. Then differentiating, factoring and substituting for $L$ the second order equation

$$
\begin{aligned}
r^{\prime \prime} & =L+4 a r-3 b r^{2} \\
& =\left(\left(r^{\prime}\right)^{2}+4 T_{0}^{2}\right) /(2 r)+2 r^{2},
\end{aligned}
$$

equivalent to the system (3.1-2) and the integrals (3.4), is the outcome if the constants $a$ and $b$ are chosen to be

$$
a=\kappa \gamma_{\perp} v_{0} \quad \text { and } \quad b=\gamma_{\perp} \gamma_{\|} / 2 .
$$

So the system (3.1-3) can be replaced by the pair

$$
\begin{aligned}
s^{2} & =2 L r-4 T_{0}^{2}+4 a r^{2}-2 b r^{3}, \\
s^{\prime} & =L+4 a r-3 b r^{2},
\end{aligned}
$$

and it is convenient to do so. This is the case despite the unorthodox feature of the latter whereby what is, in effect, an integration constant of (3.9) occurs as a coefficient. For with $a$ and $b$ fixed, $T_{0}$ and say $r(s=0)$ given, as in an orthodox initial value problem, the constant $L$ must then be specified consistent with these values. So the initial values determine a coefficient in the equation.

Here it is remarked that the reformulation of (3.1-3) as (3.8-9) to some extent justifies the local linearization of the full system (2.25-8) at the point $\mathbf{C}$ discussed in the previous section - there are no overtly singular terms in (3.8-9) at the field singularity, in contrast with (2.26) and (3.2).

Consider (3.8), and recall that $r$ (the square of a real variable) must be positive. Solutions can only exist for its right hand side positive on some interval $r \geq 0$, and since the coefficient $b$ is strictly positive then, by Descartes' rule of signs:

if $T_{0} \neq 0$, there are no more than two roots of $(s(r))^{2}$ in $r>0$, and as $(s(0))^{2}<0$, if one, then two;

and if $T_{0}=0$, then

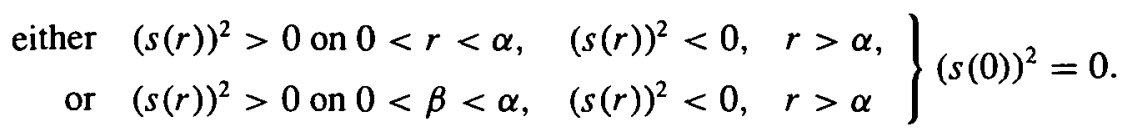


Thus, if solutions of the system (3.8-9) exist at all, they are oscillatory.

Although it is tempting to indulge in the legerdemain of Jacobian Elliptic functions in the solution of (3.8), the calculations are more straightforward if a direct line is taken. If $L$ were known, all such solutions could be expressed in terms of an amplitude parameter $\Delta$ (unrelated to the physical parameter $\hat{\Delta}$ ), and angular frequency $\hat{\lambda}(L, a, b, \Delta)$

$$
\begin{aligned}
r(\tau) & =\hat{\rho}(L, a, b, \Delta, \hat{\lambda} \tau) \\
& =\hat{\rho}_{0}(L, a, b)+\Delta \sum_{0}^{\infty} \Delta^{|n-1|} \hat{a}_{n}(L, a, b, \Delta) \cos (n \hat{\lambda} \tau),
\end{aligned}
$$

where

$$
\hat{a}_{1}=1,
$$

and the other $\hat{a}_{n}$ and $\hat{\lambda}^{2}$ are analytic functions of $\Delta^{2}$ whose radius of convergence need not be small. The $\Delta$-independent part of the mean is

$$
\hat{\rho}_{0}=\left(2 a+\left(4 a^{2}+3 L b\right)^{1 / 2}\right) /(3 b)
$$

and the coefficient of $\Delta^{0}$ in the power (Taylor) series expansion of $\hat{\lambda}^{2}$ is

$$
\hat{\lambda}_{0}^{2}=2\left(4 a^{2}+3 L b\right)^{1 / 2},
$$

the first of these (3.12) being the value of $r$ for which the right hand side of (3.9) is zero, and the second the negative of the value of the $r$-derivative of that right hand side at its zero. The constant $L$ is related to $T_{0}^{2}, a, b$ and $\Delta$ by solving

$$
\left[2 L r-4 T_{0}^{2}+4 a r^{2}-2 b r^{3}\right]_{\tau=0}=0
$$

for the function $L\left(\Delta, T_{0}^{2}, a, b\right)$. As might be anticipated, the behaviour of $L$ depends strongly on the sign of the parameter $a$. The solution of (3.14) is discussed in Section 6 in (what turns out as) the relevant case $T_{0}=0$.

If the last described operation is executed, it can be assumed that

$$
\begin{aligned}
\hat{\rho}(L, a, b, \Delta ; \hat{\lambda} \tau) & =\rho\left(T_{0}, a, b, \Delta ; \lambda \tau\right) \\
& \equiv \rho_{0}+\Delta \sum_{0}^{\infty} \Delta^{|n-1|} a_{n} \cos (n \lambda \tau)
\end{aligned}
$$

and

$$
\hat{\lambda}(L, a, b, \Delta)=\lambda\left(T_{0}^{2}, a, b, \Delta\right)
$$

are known functions of the parameters $\left(T_{0}^{2}, a, b, \Delta\right)$. It is useful to observe that $r(\psi)=\rho\left(T_{0}^{2}, a, b, \Delta ; \psi\right)$ satisfies the system

$$
\begin{aligned}
& \lambda \frac{d r}{d \psi}=s, \\
& \lambda \frac{d s}{d \psi}=\left(s^{2}+4 T_{0}^{2}\right) /(2 r)+2 a r-2 b r^{2} .
\end{aligned}
$$




\section{The varied system}

The nonlinear system (3.1-3), now re-expressed as

$$
\begin{array}{ll}
\lambda \frac{d r}{d \psi}=s, & \lambda \frac{d s}{d \psi}=\left(s^{2}+4 T_{0}^{2}\right) /(2 r)+2 \kappa \gamma_{\perp} v_{0} r-2 b r^{2}, \\
\lambda \frac{d T_{0}}{d \psi}=0, & \lambda \frac{d v_{0}}{d \psi}=0,
\end{array}
$$

induces a linear homogeneous system of first variation. Suppose $s=\lambda d \rho / d \psi \equiv \sigma$ and $r=\rho\left(T_{0}^{2}, 2 \kappa \gamma_{\perp} v_{0}, b ; \psi\right)$ are known solutions of the system (4.1). Then this induced system is

$$
\begin{aligned}
& \lambda \frac{d x}{d \psi}=y, \\
& \lambda \frac{d x}{d \psi}=-\left(\sigma+4 T_{0}^{2}\right) x /\left(2 \rho^{2}\right)+\left(2 \sigma y+8 T_{0} \zeta\right) /(2 \rho)+2 \kappa \gamma_{\perp} \rho \xi+2 \kappa \gamma_{\perp} v_{0} x-4 b \rho x \\
& \lambda \frac{d \xi}{d \psi}=0, \\
& \lambda \frac{d \xi}{d \psi}=0 .
\end{aligned}
$$

A solution matrix for this system is

$$
M=\left[\begin{array}{cccc}
\frac{d \rho}{d \psi} & \frac{d \rho}{d \Delta} & \frac{d \rho}{d T} & \frac{d \rho}{d v} \\
\lambda \frac{d^{2} \rho}{d \psi^{2}} & \lambda \frac{d}{d \psi}\left(\frac{d \rho}{d \Delta}\right) & \lambda \frac{d}{d \psi}\left(\frac{d \rho}{d T}\right) & \lambda \frac{d}{d \psi}\left(\frac{d \rho}{d v}\right) \\
0 & 0 & 1 & 0 \\
0 & 0 & 0 & 1
\end{array}\right],
$$

the matrix entries being evaluated at $T_{0}, v_{0}$, and $\Delta$. The matrix entries formally denoted as $d \rho / d \Delta$ etc. are in fact

$$
\begin{aligned}
& \frac{d \rho}{d \Delta}=\frac{\partial \rho_{0}}{\partial \Delta}-\frac{\Delta \psi}{\lambda} \frac{\partial \lambda}{\partial \Delta} \sum_{1}^{\infty} n a_{n} \Delta^{|n-1|} \sin (n \psi)+\sum_{0}^{\infty} \frac{\partial}{\partial \Delta}\left(\Delta^{|+| n-1 \mid} a_{n}\right) \cos (n \psi), \\
& \frac{d \rho}{d T}=\frac{\partial \rho_{0}}{\partial T}-\frac{\Delta \psi}{\lambda} \frac{\partial \lambda}{\partial T} \sum_{1}^{\infty} n a_{n} \Delta^{|n-1|} \sin (n \psi)+\Delta \sum_{0}^{\infty} \Delta^{|n-1|} \frac{\partial}{\partial T}\left(a_{n}\right) \cos (n \psi), \\
& \frac{d \rho}{d v}=\frac{\partial \rho_{0}}{\partial v}-\frac{\Delta \psi}{\lambda} \frac{\partial \lambda}{\partial v} \sum_{1}^{\infty} n a_{n} \Delta^{|n-1|} \sin (n \psi)+\Delta \sum_{0}^{\infty} \Delta^{|n-1|} \frac{\partial}{\partial v}\left(a_{n}\right) \cos (n \psi)
\end{aligned}
$$


where $\rho_{0}$ and $a_{n}$ are assumed known functions of $T, v$ and $\Delta$. All these are even functions of $\psi$ on $[-\pi, \pi]$ and, indicative of the exceptional case of Floquet theory, all contain a term proportional to

$$
\psi \frac{d \rho}{d \psi}=-\Delta \psi \sum_{0}^{\infty} n a_{n} \Delta^{|n-1|} \sin (n \psi) .
$$

The inverse $M^{-1}$ of the solution matrix is needed, and since $M$ partitions into four $2 \times 2$ submatrices

$$
M=\left[\begin{array}{ll}
A & B \\
0 & I
\end{array}\right],
$$

then its inverse is

$$
M^{-1}=\left[\begin{array}{cc}
A^{-1} & -A^{-1} B \\
0 & I
\end{array}\right] .
$$

In calculating $A^{-1}$, the factor $(\operatorname{det} A)^{-1}$ can be calculated from the Wronskian relationship

$$
\operatorname{det} A \propto \exp \left(\int \text { trace of the coefficient matrix of (4.2) }\right)
$$

and so, referring to (4.2) (the trace is $\left.2 \sigma y /(2 \rho \lambda)=\rho^{-1} d \rho / d \psi\right)$,

$$
\operatorname{det} A \propto \rho,
$$

and the inverted submatrix is

$$
A^{-1} \propto \rho^{-1}\left[\begin{array}{cc}
\lambda \frac{d}{d \psi}\left(\frac{d \rho}{d \Delta}\right) & -\frac{d \rho}{d \Delta} \\
-\lambda \frac{d^{2} \rho}{d \psi^{2}} & \frac{d \rho}{d \psi}
\end{array}\right] .
$$

The fourth column of $M^{-1}$ which will be required explicitly is proportional to

$$
\rho^{-1}\left[\begin{array}{c}
-\lambda \frac{d \rho}{d v} \frac{d}{d \psi}\left(\frac{d \rho}{d \Delta}\right)+\lambda \frac{d \rho}{d \Delta} \frac{d}{d \psi}\left(\frac{d \rho}{d v}\right) \\
\lambda \frac{d^{2} \rho}{d \psi^{2}} \frac{d \rho}{d v}-\lambda \frac{d \rho}{d \psi} \frac{d}{d \psi}\left(\frac{d \rho}{d v}\right) \\
0 \\
1
\end{array}\right]
$$

The first and second entries of the vector (4.8) are respectively odd and even functions of $\psi$ on $[-\pi, \pi]$. 


\section{Application of the extended Kuzmak method}

Now revert to the problem of synthesizing approximations to the solutions of (2.2528) by an extension of the method of Kuzmak [7]. The first step is to invoke two time scales for the problem: a slow one

$$
t=\epsilon \tau,
$$

that is, the original $t$ of the problem resurrected (the derivative with respect to which being again denoted by ${ }^{\circ}$ where unambiguous), and a fast one

$$
\psi=\epsilon^{-1} \phi(t)=\epsilon^{-1} \sum_{0}^{\infty} \epsilon^{n} \phi_{n}(t) .
$$

This last is an elaboration of the Kuzmak [7] algorithm; its purpose is to allow for more general perturbations - specifically those which are even in the fast time scale - should they occur in the calculations.

Then (2.25-28) for $r(\psi, t)$ etc. are formally

$$
\begin{aligned}
& \dot{\phi} \frac{\partial r}{\partial \psi}+\epsilon \frac{\partial r}{\partial t}=s \\
& \dot{\phi} \frac{\partial s}{\partial \psi}+\epsilon \frac{\partial s}{\partial t}=\left(s^{2}+4 T^{2}\right) /(2 r)-\epsilon(p s-2 q T)+2 \kappa \gamma_{\perp} v r-2 b r^{2} \\
& \dot{\phi} \frac{\partial T}{\partial \psi}+\epsilon \frac{\partial T}{\partial t}=-\epsilon(p T+q s / 2), \\
& \dot{\phi} \frac{\partial v}{\partial \psi}+\epsilon \frac{\partial v}{\partial t}=-\epsilon \gamma_{\|}\left(v-1+\left(1-\gamma_{\|} /(2 \kappa)\right) r\right) .
\end{aligned}
$$

In essence, Kuzmak's method is to construct formal approximate solutions of these equations that are periodic in the fast variable $\psi$.

The equations governing the leading terms of formal expansions in powers of $\epsilon$ of the dependent variables are

$$
\begin{aligned}
& \dot{\phi}_{0} \frac{\partial r_{0}}{\partial \psi}=s_{0}, \\
& \dot{\phi}_{0} \frac{\partial s_{0}}{\partial \psi}=\left(s_{0}^{2}+4 T_{0}^{2}\right) /\left(2 r_{0}\right)+2 \kappa \gamma_{\perp} v_{0} r_{0}-2 b r_{0}^{2} \\
& \dot{\phi}_{0} \frac{\partial T_{0}}{\partial \psi}=0 \\
& \dot{\phi}_{0} \frac{\partial v_{0}}{\partial \psi}=0
\end{aligned}
$$


and those governing the next are

$$
\begin{aligned}
& \dot{\phi}_{0} \frac{\partial r_{1}}{\partial \psi}= s_{1}-\frac{\partial r_{0}}{\partial t}-\dot{\phi}_{1} \frac{\partial r_{0}}{\partial \psi}, \\
& \dot{\phi}_{0} \frac{\partial s_{1}}{\partial \psi}=-\left(s_{0}^{2}+4 T_{0}^{2}\right) r_{1} /\left(2 r_{0}\right)^{2}+\left(2 s_{0} s_{1}+8 T_{0} T_{1}\right) /\left(2 r_{0}\right)+2 \kappa \gamma_{\perp} v_{0} r_{1} \\
& \quad+2 \kappa \gamma_{\perp} r_{0} v_{1}-4 b r_{0} r_{1}-\left(p s_{0}-2 q T_{0}\right)-\frac{\partial s_{0}}{\partial t}-\dot{\phi}_{1} \frac{\partial s_{0}}{\partial \psi}, \\
& \dot{\phi}_{0} \frac{\partial T_{1}}{\partial \psi}=-\left(p T_{0}+q s_{0} / 2\right)-\frac{\partial T_{0}}{\partial t}-\dot{\phi}_{1} \frac{\partial T_{0}}{\partial \psi}, \\
& \dot{\phi}_{0} \frac{\partial v_{1}}{\partial \psi}=-\gamma_{\|}\left(v_{0}-1+\left(1-\gamma_{\|} / 2 \kappa\right) r_{0}\right)-\frac{\partial v_{0}}{\partial t}-\dot{\phi}_{1} \frac{\partial v_{0}}{\partial \psi} .
\end{aligned}
$$

Equations (5.5-8) have $\psi$-periodic solutions expressible in terms of the functions introduced in Section 3,

$$
\begin{aligned}
r_{0}(\psi, t) & =\rho\left(T_{0}^{2}, a, b, \Delta ; \psi\right), \\
s_{0}(\psi, t) & =\lambda \frac{\partial \rho}{\partial \psi}, \\
\dot{\phi}_{0}(t) & =\lambda\left(T_{0}^{2}, a, b, \Delta\right) \equiv \cdot \lambda,
\end{aligned}
$$

and it is also inferred from $(5.5-8)$ that

$$
T_{0}(t), \quad a=\kappa \gamma_{\perp} v_{0}(t), \quad \Delta(t),
$$

are independent of $\psi$, but have otherwise to be determined, for $b=\gamma_{\perp} \gamma_{\|} / 2$. The four unknowns $\dot{\phi}_{1}(t), T_{0}(t), v_{0}(t)$ and $\Delta(t)$ are found by requiring (Kuzmak [7]) the solutions $r_{1}, s_{1}, T_{1}$ and $v_{1}$ also to be periodic in $\psi$, and this will be so if the four conditions

$$
\int_{-\pi}^{\pi} M^{-1}\left[\begin{array}{c}
\frac{\partial r_{0}}{\partial t}+\dot{\phi}_{1} \frac{\partial r_{0}}{\partial \psi} \\
\left(p s_{0}+2 q T_{0}\right)+\frac{\partial s_{0}}{\partial t}+\dot{\phi}_{1} \frac{\partial s_{0}}{\partial \psi} \\
\left(p T_{0}+q s_{0} / 2\right)+\frac{d T_{0}}{d t} \\
\gamma_{\|}\left(v_{0}-1+\left(1-\gamma_{\|} / 2 \kappa\right) r_{0}\right)+\frac{d v_{0}}{d t}
\end{array}\right] d \psi\left(\dot{\phi}_{0}\right)^{-1}=\mathbf{0}
$$

are satisfied. The entries in the vector above are the non-homogeneous terms in (5.912), simplified by the partial solutions (5.16) which show $T_{0}$ and $v_{0}$ to be independent of $\psi$. Thus some terms vanish identically, and partial $t$-derivatives of $T_{0}$ and $v_{0}$ are replaced with ordinary $t$-derivatives. 
The third row $(0,0,1,0)$ of the matrix $M^{-1}$ leads to an immediate result. This component of the condition (5.17),

$$
\int_{-\pi}^{\pi}\left(p T_{0}+q s_{0} / 2+\frac{d T_{0}}{d t}\right) d \psi=0
$$

reduces because $s_{0}(\psi, t)$ is an odd function of $\psi$ on $[-\pi, \pi]$ and its integral over that interval therefore vanishes. Thus $T_{0}(t)$ must satisfy the differential equation

$$
p T_{o}+\frac{d T_{0}}{d t}=0
$$

and so

$$
T_{0}=\text { constant } \exp (-p t)=K \exp \left(-\left(\kappa+\gamma_{\perp}\right) t\right) .
$$

The constant $K$ is determined by initial conditions, but if solutions are sought evolving from large negative $t$, or if asymptotic states are considered, then the natural reduction is

$$
T_{0}(t)=0
$$

and it is adopted for what follows.

With this assumption (5.19), the matrix $M$ is significantly simplified. Recalling that the coefficient in (3.1-2) defining $\rho$ is $T_{0}$, and the equations are not generally singular when $T_{0}=0$, then both

$$
\frac{\partial \rho}{\partial T} \quad \text { and } \quad \frac{d}{d \psi}\left(\frac{d \rho}{d T}\right)
$$

evaluated at $T_{0}=0$ are identically zero, so the third column of $M$ is now

$$
\mathbf{M}_{3}=\left[\begin{array}{l}
0 \\
0 \\
1 \\
0
\end{array}\right]
$$

From the structure of $M$, the third column of $M^{-1}$ is also $\mathbf{M}_{3}$, so the third element of the vector factor (potentially only the term $q s_{0} / 2$ ) in the integrand (5.17) takes no further part in proceedings.

The fourth condition (5.17) is

$$
\int_{-\pi}^{\pi}\left[\gamma_{\|}\left(v_{0}-1+\left(1-\gamma_{\|} /(2 \kappa)\right) r_{0}\right)+\frac{d v_{0}}{d t}\right] d \psi=0
$$


By referring to the solution (5.13), and the evaluation (3.15) of $\rho$, it will be seen that if the $\psi$-period mean of $\rho(0, a, b, \Delta ; \psi)$ is defined to be

$$
\bar{\rho}=\rho_{0}+\Delta^{2} a_{0}
$$

then this condition is

$$
\frac{d v_{0}}{d t}+\gamma_{\|}\left(v_{0}-1+\left(1-\gamma_{\|} /(2 \kappa)\right) \bar{\rho}\right)=0
$$

This last result will later be used in the form

$$
\frac{d v_{0}}{d t}+\gamma_{\|}\left(v_{0}-1+\left(1-\gamma_{\|} /(2 \kappa)\right) r_{0}\right)=\gamma_{\|}\left(1-\gamma_{\|} /(2 \kappa)\right)(\rho-\bar{\rho}) .
$$

The first condition (5.17) contracts because of the parity of the terms in the integral. Thus it follows that, on omitting terms which vanish by their parity property, one obtains

$$
\int_{-\pi}^{\pi} \dot{\phi}_{1}\left(\lambda \frac{d}{d \psi}\left(\frac{d \rho}{d \Delta}\right) \frac{d \rho}{d \psi}-\frac{d \rho}{d \Delta} \lambda \frac{d^{2} \rho}{d \psi^{2}}\right) d \psi=\int_{-\pi}^{\pi} 2 \dot{\phi}_{1} \lambda \frac{d}{d \psi}\left(\frac{d \rho}{d \Delta}\right) \frac{d \rho}{d \psi} d \psi=0
$$

after integrating by parts. Since $\phi_{1}$ is independent of $\psi$, then it follows that this integral will vanish in general only if

$$
\dot{\phi}_{1}=0 \text {. }
$$

After some manipulation, the second condition (5.17) reduces to

$$
\begin{aligned}
& \left(\frac{d}{d t}+p+\frac{1}{\lambda} \frac{d \lambda}{d t}\right)\left(\int_{-\pi}^{\pi} \rho^{-1}\left(\frac{d \rho}{d \psi}\right)^{2} d \psi\right) \\
& =-\gamma_{\|}\left(1-\gamma_{\|} /(2 \kappa)\right) \int_{-\pi}^{\pi}\left(2 \rho^{-1}(\rho-\bar{\rho}) \frac{d^{2} \rho}{d \psi^{2}}+\bar{\rho} \rho^{-2}\left(\frac{d \rho}{d \psi}\right)^{2}\right) \frac{d \rho}{d v} d \psi,
\end{aligned}
$$

and this, and (5.21) are the conditions required to determine the remaining unknowns $\Delta(t)$ and $v_{0}(t)$.

Two simplifications can be made in (5.24a). The term $\lambda_{-1} d \lambda / d t$ can be omitted from its left hand side operator if both the operand and the right hand side are multiplied by $\lambda$, and so it becomes

$$
\begin{aligned}
& \left(\frac{d}{d t}+p\right)\left(\lambda \int_{-\pi}^{\pi} \rho^{-1}\left(\frac{d \rho}{d \psi}\right)^{2} d \psi\right) \\
& =-\gamma_{\|}\left(1-\gamma_{\|} /(2 \kappa)\right) \lambda \int_{-\pi}^{\pi}\left(2 \rho^{-1}(\rho-\bar{\rho}) \frac{d^{2} \rho}{d \psi^{2}}+\bar{\rho} \rho^{-2}\left(\frac{d \rho}{d \psi}\right)^{2}\right) \frac{d \rho}{d v} d \psi .
\end{aligned}
$$


Also, and more significantly, the structure of the factor $d \rho / d v(4.4-5)$ allows a reduction in calculation of the right hand side of (5.24). This factor contains a term which is the product of slowly varying factors with $\psi d \rho / d \psi$; integrating by parts the term containing the second derivative shows that

$$
\begin{aligned}
\int_{-\pi}^{\pi}\left(2 \rho^{-1}(\rho-\bar{\rho}) \frac{d^{2} \rho}{d \psi^{2}}\right. & \left.+\bar{\rho} \rho^{-2}\left(\frac{d \rho}{d \psi}\right)^{2}\right) \frac{d \rho}{d \psi} \psi d \psi \\
& =-\int_{-\pi}^{\pi} \rho^{-1}(\rho-\bar{\rho})\left(\frac{d \rho}{d \psi}\right)^{2} d \psi
\end{aligned}
$$

(The integrated part vanishes because $d \rho / d \psi$ does at the integration terminals.)

Equations (5.21) and (5.24) together then control the evolution of the two slow variables $v_{0}$ and $\Delta$. There is an obvious further reduction; if the two equations are expressed as

$$
\frac{d v_{0}}{d t}=J\left(\Delta, v_{0}\right)
$$

and

$$
\frac{d}{d t} G\left(\Delta, v_{0}\right)=\frac{\partial G}{\partial \Delta} \frac{d \Delta}{d t}+\frac{\partial G}{d v_{0}} \frac{d v_{0}}{d t}=H\left(\Delta, v_{0}\right),
$$

then the first order system describing a phase plane

$$
\frac{d \Delta}{d v_{0}}=\frac{H-J \partial G / \partial v_{0}}{J \partial G / \partial \Delta}
$$

is the result. Thus the underlying dynamics of the Kuzmak approach to this problem has produced what is, in effect, a description of an abstract Poincare section of the trajectories of the system (5.1-4), or the system (2.25-28) sampled at local minima of $r$. That this is the case follows from the construction. It determines the slow modulation of $\Delta$ and $v_{0}$ by (condition (5.17)) forcing fast variable $(\psi)$ periodicity on approximations to $r$ and $v$, sampling them at $2 \pi$ intervals at points that are also local minima of the approximation to $r$.

\section{Calculations}

The Fourier representation (3.10) of the solution of (3.6) is first calculated. The Fourier coefficients $a_{n}$ are all analytic in powers of $\Delta^{2}$ so the calculations are routine; but here the calculation is only taken to $\Delta^{3}$. Recall that the asymptotic state $T_{0}=0$ only is considered.

If

$$
\eta=r-\rho_{0}
$$


where $r=\rho_{0}$ is the zero of the right hand side of (3.6), and is evaluated in (3.12), then (3.6) can be expressed as

$$
\begin{aligned}
\eta^{\prime \prime} & =-2\left(4 a^{2}+3 L b\right)^{1 / 2} \eta-3 b \eta^{2} \\
& \equiv-P \eta-Q \eta^{2},
\end{aligned}
$$

say. The Fourier representation of the solution of the last equation, correct to $O\left(\Delta^{3}\right)$ in the amplitude parameter $\Delta$ is

$$
\begin{aligned}
\eta(\lambda \tau)= & \Delta \cos (\hat{\lambda} \tau)+\Delta^{2}\{-(Q /(2 P))+(Q /(6 P)) \cos (2 \hat{\lambda} \tau)\} \\
& +\Delta^{3}\left(Q^{2} /\left(48 P^{2}\right) \cos (3 \hat{\lambda} \tau)+O\left(\Delta^{4}\right),\right. \\
\hat{\lambda}^{2}= & P\left(1-5 \Delta^{2} Q^{2} /\left(6 P^{2}\right)\right)+O\left(\Delta^{4}\right), \\
\hat{\lambda} & =P^{1 / 2}\left(1-5 \Delta^{2} Q^{2} /\left(12 P^{2}\right)\right)+O\left(\Delta^{4}\right)
\end{aligned}
$$

provided $P$ is not small. The coefficients of $\cos (0 \tau), \cos (2 \tau)$ and $\cos (3 \tau)$ are respectively the leading terms in the expansions in powers of $\Delta^{2}$ of $a_{0}, a_{2}$ and $a_{3}$.

The calculation of $L(\Delta)$ can now be made. Attention is restricted to the asymptotic state $T_{0}=0$ in (3.14), as foreshadowed in Section 5. In essence, $L$ must be chosen so that the two statements

$$
r_{\max }=\rho_{0}+\Delta \sum_{0}^{\infty} \Delta^{|n-1|} a_{n}
$$

and

$$
r_{\max }=\left(a+\left(a^{2}+L b\right)^{1 / 2}\right) / b
$$

are compatible. Equation (6.4) is the evaluation of (3.10) at $\tau=0$, and (6.5) is the location of the largest zero of (3.14) when $T_{0}=0$. As remarked earlier the evaluation of $L$ is strongly influenced by the sign of $a$.

Recalling the evaluation (3.12) of $\hat{\rho}_{0},(6.4-5)$ require that

$$
3\left(a^{2}+L b\right)^{1 / 2}-\left(4 a^{2}+3 L b\right)^{1 / 2}=-a+3 b \Delta+3 b \Delta^{2}\left(a_{0}+a_{2}\right)+O\left(\Delta^{3}\right) .
$$

This equation can be solved for $L(\Delta)$, depending on the sign of $a$. Solutions are: when $a<O$, both $\{|a|, 1 \gg \Delta\}$ and

$$
L(\Delta)=4|a| \Delta+8 b \Delta^{2}+O\left(\Delta^{3}\right),
$$

and when both $\{a, 1 \gg \Delta>0\}$

$$
L(\Delta)=-\left(a^{2} / b\right)+b \Delta^{2}+O\left(\Delta^{3}\right) .
$$

When $|a| \sim O(\Delta), L$ is continuous and $O\left(\Delta^{2}\right)$ but series representation of $L(\Delta)$ converges only slowly. This is because $P$ becomes $O(\Delta)$, and the coefficients in 
the Fourier representation (3.10) decrease arithmetically only - that is, unassisted by powers of $\Delta$ when $\Delta$ is small. (The calculation of the Fourier coefficients is not essentially restricted to small $\Delta$, but approximations using short truncations of power series are only useful if it is.)

The critical surd which recurs through the calculations can now be expressed independently of $L$. When $|a|, 1 \gg \Delta$, and $a<0$ it is

$$
\left(4 a^{2}+3 L b\right)^{1 / 2}=2|a|\left(1+3 b \Delta /(2|a|)+O\left(\Delta^{2}\right)\right)
$$

and when $a, 1 \gg \Delta$ it is

$$
\left(4 a^{2}+3 L b\right)^{1 / 2}=a\left(1+3 b^{2} \Delta^{2} /\left(2 a^{2}\right)+O\left(\Delta^{3}\right)\right) .
$$

Corresponding evaluations of $\rho_{0}$ are

$$
\begin{array}{ll}
(a<0): & \rho_{0}=\Delta+O\left(\Delta^{2}\right), \\
(a>0): & \rho_{0}=\left(3 a+3 b^{2} \Delta^{2} /(2 a)+O\left(\Delta^{3}\right)\right) /(3 b) .
\end{array}
$$

The lowest order approximations to $\rho$ and $\lambda$ can be used to check (5.21) and (5.24) against linear stability theory. Suppose $a>0$; then, correct to $O(\Delta)$, we have

$$
\begin{aligned}
\rho=(a / b)+\Delta \cos (\psi) & =\left(2 \kappa v_{0} / \gamma_{\|}\right)+\Delta \cos (\psi), \\
\rho_{0}, \bar{\rho} & =\left(2 \kappa v_{0} / \gamma_{\|}\right),
\end{aligned}
$$

so (5.21) is approximated as

$$
\frac{d v_{0}}{d t}+\gamma_{\|}\left(v_{0}-1\right)+\gamma_{\|}\left(1-\gamma_{\|} /(2 \kappa)\right)\left(2 \kappa v_{0} / \gamma_{\|}\right)=\frac{d v_{0}}{d t}+2 \kappa v_{0}-\gamma_{\|}=0
$$

which indicates that $v_{0}=\left(\gamma_{\|} / 2 \kappa\right)$ is a stable fixed point with eigenvalue $(-2 \kappa)$. Similarly, with (3.13) $\lambda$ approximated by the truncated approximation to

$$
\lambda_{0}=(2 a)^{1 / 2}+O\left(\Delta^{2}\right)
$$

and $\rho^{-1}$ and $\left(\bar{\rho} \rho^{-2}\right)$ both approximated as $\rho_{0}^{-1},(5.24)$ gives

$$
\left(\frac{d}{d t}+p\right)\left(\Delta^{2} / v_{0}^{1 / 2}\right)=\left(2 \kappa-\gamma_{\|}\right)\left(\Delta^{2} / v_{0}^{1 / 2}\right) .
$$

Recalling $p=\kappa+\gamma_{\perp}$, this gives the eigenvalue $\left(-\kappa+\gamma_{\|}+\gamma_{\perp}\right.$ ) (for $\Delta^{2}$, twice the eigenvalue for $\Delta$ ) at the fixed point $\Delta=0$ (that is, $r=1$ ). So, as must be the case, the Kuzmak theory is consistent with linear local analysis stated at the end of Section 2 for the fixed point $\mathbf{D}$ - recall that $(2 a)^{1 / 2}=\left(\gamma_{\|} \gamma_{\perp}\right)^{1 / 2}$ and note the eigenvalue $(-p)$ from the $T_{0}(5.18)$ - when $v_{0}$ (or $a$ ) is positive. 
If, on the other hand, an initial condition has $v_{0}$ negative (but $\left|v_{0}\right|$ not small) then, on substituting for $\bar{\rho} \cong \rho_{0}(6.8)$ in (5.21) it is seen that $v_{0}$ evolves as

$$
\frac{d v_{0}}{d t}+\gamma_{\|}\left(v_{0}-1+\left(1-\gamma_{\|} /(2 \kappa)\right) \Delta\right)=0
$$

and since (again for $v_{0}$ negative) $d \rho / d v=0$, then (5.24) reduces to

$$
\left(\frac{d}{d t}+p\right)\left(\left|v_{0}\right|^{1 / 2} \Delta\right)=0
$$

These last two equations taken together imply (since $p$ is positive) that $v_{0} \rightarrow 0$ at some finite $t$ and the analysis fails. It seems reasonable to interpret this as indicating a sign change for $v_{0}$ so that the $v_{0}>0$ analysis applies. Considerations are now restricted to the latter case.

For making higher order calculations, truncations are made appropriately and one has

$$
\begin{array}{ccc}
a_{0}=-3 b /(4 a) ; \quad a_{2}=b /(4 a) ; & \lambda=(2 a)^{1 / 2}\left(1-3 b^{2} \Delta^{2} /\left(16 a^{2}\right)\right) \\
\rho_{0}=a\left(1+b^{2} \Delta^{2} /\left(2 a^{2}\right)\right) / b ; & \bar{\rho}=a\left(1-b^{2} \Delta^{2} /\left(4 a^{2}\right)\right) / b .
\end{array}
$$

It is convenient to introduce a parameter combination

$$
c=\gamma_{\|} /(2 \kappa)
$$

so that

$$
b / a=c / v_{0} .
$$

The calculations required to take (5.24) into the form (5.25) to $O\left(\Delta^{4}\right)$ are tedious, but routine. If some obvious parameter and constant cancellations are made, then the truncations are

$$
\begin{aligned}
G\left(\Delta, v_{0}\right) & =\Delta^{2} v_{0}^{1 / 2}\left(1+3 c^{2} \Delta^{2} /\left(16 v_{0}^{2}\right)\right), \\
H\left(\Delta, v_{0}\right) & =\left(2 \kappa-\gamma_{\|}\right) \Delta^{2} v_{0}^{-1 / 2}\left(1+c^{2} \Delta^{2} /\left(4 v_{0}^{2}\right)\right)-p G\left(\Delta, v_{0}\right) \\
& =\Delta^{2} v_{0}^{-1 / 2}\left(\left(\kappa-\gamma_{\|}-\gamma_{\perp}\right)+\left(3\left(\kappa-\gamma_{\|}-\gamma_{\perp}\right)+2 \kappa-\gamma_{\|}\right) c^{2} \Delta^{2} /\left(16 v_{0}^{2}\right)\right), \\
J\left(\Delta, v_{0}\right) & =\gamma_{\|}\left(1-v_{0} / c+(1-c) c \Delta^{2} /\left(4 v_{0}\right)\right) \\
& =\gamma_{\|}\left(c^{2} / 4-\left(v_{0}-c / 2\right)^{2}+(1-c) c^{2} \Delta^{2} / 4\right) /\left(c v_{0}\right) .
\end{aligned}
$$

Since the coefficient $\partial G / \partial \Delta$ is positive in the interior of the first quadrant of the $\left(v_{0}, \Delta\right)$ plane, the direction field associated with (5.25) has singular points (in particular) at the intersections, if any, of the contours $J=0$ and $H=0$. If $c<1$ (that is, $2 \kappa>\gamma_{\|}$), $J=0$ is a half branch of a hyperbola in the interior of the first quadrant; if $c>1$, 
it is the quadrant of an ellipse. In each case the conic section is centered at $(c / 2,0)$, and its intersection with $\Delta=0$ locates the fixed point $(c, 0)$ whose bifurcation is of interest. The contour $H=0$ is the one of the lines obtained from solving

$$
16 v_{0}^{2}=-\left(3+\left(2 \kappa-\gamma_{\|}\right) /\left(\kappa-\gamma_{\|}-\gamma_{\perp}\right)\right) c^{2} \Delta^{2}
$$

and a real line is available only if the right hand side coefficient is positive. Thus there is a restriction to the region in parameter space for which an asymptotic bifurcated solution is available. If $\left(\kappa-\gamma_{\|}-\gamma_{\perp}\right)$ is small, then $\left(2 \kappa-\gamma_{\|}\right)$is positive $(c<1)$ and not small. Then $\left(\kappa-\gamma_{\|}-\gamma_{\perp}\right)$ can always be chosen sufficiently small and negative that the right hand side coefficient in (6.14) is positive and large enough that the contour $H=0$ intersects the right, top half branch of the hyperbola $J=0$. Call this intersection $F$.

If, on the other hand, $2 \kappa-\gamma_{\|}$is negative, then $\left(\kappa-\gamma_{\|}-\gamma_{\perp}\right)$ must be negative and $H$ is positive in the interior of the first quadrant of the $\left(v_{0}, \Delta\right)$ plane, so there can be no fixed points in the neighbourhood of $(c, 0)$. (In this case $\left(\kappa-\gamma_{\|}-\gamma_{\perp}\right)$ cannot be small as the parameters are assumed not to be small, so the question of bifurcation does not really arise.)

When the fixed point $F$ exists, the local linearization of (5.25) can be computed, at least for small $\Delta$. Routine calculations show that the flow is a saddle, with its unstable manifold having large positive slope, and its stable manifold having small positive slope. The fixed point $F$ represents the subcritically bifurcated solution in the original equations (2.1-3). This result, namely that the bifurcation is only subcritical when possible, is the same as that for the real Lorenz equations (see for example Weiss and Vilaseca [8]) but it is at variance with the property found by Fowler, Gibbon and McGuinness [4] for the complex Lorenz equations where both sub- and supercritical bifurcations can occur for different parameter combinations.

\section{An outline of a proof}

The present writer is unaware of any literature which discusses the validity limitations of the Kuzmak algorithm. An argument along the following lines may suffice. The system whose solution is approximated $(5.1-4)$ can be written in vector notation (but suppressing explicit slow variation)

$$
\dot{\phi} \frac{d \mathbf{Y}}{d \psi}=\mathbf{K}(\mathbf{Y})+\epsilon h(\mathbf{Y})
$$

and the Kuzmak algorithm constructs an approximation $\mathbf{Z}$ to $\mathbf{Y}$ which satisfies

$$
\dot{\phi} \frac{d \mathbf{Z}}{d \psi}=\mathbf{K}(\mathbf{Z})+\epsilon h(\mathbf{Z})+\epsilon \dot{\phi} \mathbf{q}(\psi),
$$


where the components of $\epsilon \mathbf{q}$ are known, periodic functions of $\psi$ having a mean of $O\left(\epsilon^{2}\right)$ with respect to that variable, and uniformly bounded on intervals of $\psi$ on which both the dependent variable $r$, and $\dot{\phi}$, are bounded away from zero. On such intervals, a Lipschitz "constant" $L(t)$ can be assigned to $\dot{\phi}^{-1}(\mathbf{K}+\epsilon \mathbf{h})$ so that, in the 1-norm, and pointwise, the error $E$ satisfies the inequality of the form

$$
\|Z-Y\|(\psi)=E(\psi) \leq \int L E d \psi+\epsilon \int Q d \psi .
$$

The integrand $Q$ is arrived at as follows. The components of $\epsilon \dot{\phi} \mathbf{q}$ are examined separately, and the second Mean Value Theorem of the integral calculus (Jeffreys and Jeffreys [6]) applied to their integrals. With respect to their fast variation they are integrable, and their slow variation will be a factor with bounded variation. Hence the second MVT applies, and with it a 1-norm bound on these terms which, for immediate convenience, is written as $\epsilon \int Q d \psi$. The solution of (7.3) (see Coddington and Levinson [2]) is $(\ell=\max (L))$

$$
E(\psi) \leq \epsilon \exp (\ell \psi) \int Q d \psi
$$

This suggests that the approximations supplied by (5.13) and (5.15), with $\Delta, v_{0}$ evolving as required respectively by (5.24), (5.22) and $T_{0}=0$ could be adequate only on a moderately large, but technically sufficiently large, $\psi$ interval (say $\psi \sim$ $O(\ln |\ln (\epsilon)|))$.

\section{Conclusions}

It has been shown in the preceding sections how the fifth order laser Lorenz equations (2.1-3) can be reduced (in parameter ranges where the dynamics are interesting) to a fourth order but apparently essentially only a perturbed second order system (2.2528 ). It would seem that this reduction is in any case an advantage. The variables are identified in (2.5-24).

For the classes of solution discussed, the analysis suggests that there is asymptotic locking onto the toroidal eigenfrequency $\omega$ of the quasi-steady solution (2.5-7). This conclusion follows from the asymptotic vanishing of the variable $T((5.18)$; for its relationship with the solution structure see (2.16), (2.20-1).). The fundamental parameter groupings determining solution behaviour are, in decreasing order of importance,

$$
\left(\kappa-\gamma_{\|}-\gamma_{\perp}\right), \quad\left(2 \kappa-\gamma_{\|}\right) \quad \text { and } \quad \gamma_{\|} ;
$$

the bifurcation parameter $A-A_{\text {crit }}$ acts through the scale $(\epsilon)$ of perturbations, with no other generic input. The parameters $\hat{\Delta}$ and $\delta$ have little significance for the essentials of the problem. 
In the analysis, it has been convenient to work with the slowly evolving fundamental amplitude $\Delta$, and it transpires that the asymptotic dynamics can be described in a $\left(v_{0}, \Delta\right)$ phase plane - an abstract Poincaré section of trajectories. The evaluation of $\Delta$ - presumably by Fourier analysis of individual oscillations of the variable $r-$ would not be convenient in assessment of numerical simulations, but it can be shown that $\Delta$ is an analytic function of a variable ( $m$, say) equal to the difference of the greatest and least values of $\rho$ on a cycle for the oscillations of the type described in Section 3, and this would be easily inferred from data if $r$ were identified with $\rho$. Such a variable could be used instead of $\Delta$ in a numerically generated $\left(v_{0}, m\right)$ phase plane.

It is not unreasonable to speculate that continuation to higher order of the calculations of Section 6, or numerical reconstruction of the phase plane as suggested above might lead to interesting insights. The subcritical limit cycle is determined as a fixed point in the phase plane by the intersection $F$ of the line $H=0$ with the hyperbola branch $J=0$. In the low order theory above, this requires satisfaction of the inequality

$$
(1-c)^{1 / 2}>2\left(-\left(3+\left(2 \kappa-\gamma_{\|}\right) /\left(\kappa-\gamma_{\|}-\gamma_{\perp}\right)\right)^{1 / 2}\right.
$$

$\left(c=\gamma_{\|} / 2 \kappa\right)$. Near equality, but in a higher order theory, there could be a proliferation of fixed points, and more significant dynamics. (The writer confesses that initially he had hoped to find evidence of a limit cycle in the phase plane, but so far there is none).

It is useful to examine the outcome of the method used here on the complex Lorenz model studied in [4] and [5]. The essential difference occurs at the equation corresponding to (2.27), which generally has an extra small term on its right hand side which is a constant multiple of $r$, and consequently the equation whose solution generates $T_{0}((5.18)$ above) will generally contain an extra term that is a constant multiple of $\bar{\rho}$. Such further coupling would make subsequent calculations significantly more elaborate, and the dynamics are fundamentally more complicated. This will be so as there is inherently a $\left(\Delta, v_{0}, T_{0}\right)$ space for consideration, rather than a $\left(\Delta, v_{0}\right)$ space (at least asymptotically) as above.

\section{References}

[1] F. J. Bourland and R. Haberman, "Separatrix crossing: time invariant potentials with dissipation", SIAM J. Appl. Math. 50 (1990) 1716-1744.

[2] E. A. Coddington and E. Levinson, Theory of ordinary differential equations (McGraw-Hill, New York, 1955).

[3] G. P. Flessas, "New exact solutions of the complex Lorenz equations", J. Phys. A: Math. Gen. 22 (1989) L137-L141.

[4] A. C. Fowler, J. D. Gibbon and M. J. McGuinness, "The complex Lorenz equations", Physica D 4 (1982) 139-159. 
[5] A. C. Fowler and M. J. McGuinness, "On the nature of the torus in the complex Lorenz equations", SIAM J. Appl. Math. 44 (1984) 681-700.

[6] H. Jeffreys and B. S. Jeffreys, Methods of mathematical physics, third ed. (Cambridge University Press, Cambridge, 1956).

[7] G. E. Kuzmak, "Asymptotic solutions of second order differential equations with variable coefficients", J. Appl. Math. Mech. 23 (1959) 730-744.

[8] C. O. Weiss and R. Vilaseca, The dynamics of lasers (VCH, Weinheim, 1991).

[9] H. Zeghlache, P. Mandel, N. B. Abraham and C. O. Weiss, "Phase amplitude dynamics of the laser Lorenz model", Phys. Rev. A 38 (1988) 3128-3131. 